\title{
Development and Improvement of Frequency Response Characteristic Test Tool for Broadband High Frequency Current Sensor
}

\author{
Liping Zhu 1, a, Yunyun Fu 2, b \\ North China Electric Power University, Beijing 102206, China \\ aCorresponding author:sdmyzlp@126.com, b940417119@qq.com
}

Keywords: High Frequency, Current Sensor, Improvement of Test Tool.

\begin{abstract}
According to the transmission line theory, the theoretical model of the frequency response characteristic test tool for broadband high frequency current sensor is constructed. Then, SolidWorks and XFdtd is used to model and simulate the tool to find the optimal design parameters of the core coaxial tool After the optimization is finished, the object will be made according to the drawing, and the test will be done to verify whether it is consistent with the simulation result and whether it meets the actual needs.
\end{abstract}

\section{Introduction}

With the rapid development of the national economy and the continuous improvement of the people's living standards, the society's demand for electric energy is increasing. The capacity of the power grid is expanding and the voltage level is gradually increasing. The stability and reliability of the power system are higher. Partial discharge detection is one of the common means of maintenance and repair of electrical equipment. The high frequency current sensor used in it is playing an increasingly important role in maintenance work because of its high sensitivity and good portability.

The wider the sensor band is, the more accurate the fault information can be extracted. To this end, the former scientists have developed a current sensor frequency response test system however could not achieve the effective testing of high frequency current sensor. Known current highfrequency current sensor bandwidth is up to $300 \mathrm{MHz}$, we have to design the tool not only to provide greater than $300 \mathrm{MHz}$ signal bandwidth, but also to achieve impedance matching as much as possible to reduce distortion. Therefore we use Solidworks to design and improve the shape of tool. Afterwards we use XFdtd to simulate the current sensor. In the most economical case, a tooling model with the highest current frequency can be obtained.

High-frequency measurement calibration system consists of waveform generator, high-frequency coupling tooling, R0 no-sense resistance, high-speed digital oscilloscope, control computer, control software and a variety of cable accessories. As shown below:

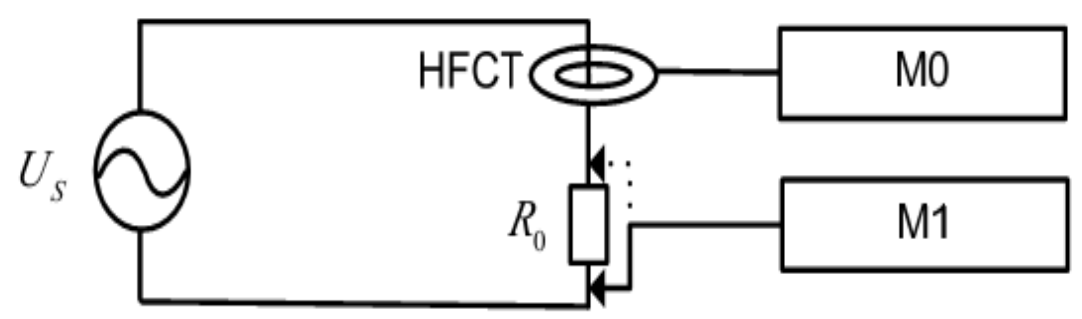

Figure 1.Test System Schematic

\section{Improvement Ideas}

At the beginning of the project, according to the relevant knowledge of impedance matching, we calculate the internal and external radius ratio of $1: 2.3$ with the aid of the impedance of the formula when the reflection is minimum.

Firstly our idea is relatively simple, we choose a rather common cylindrical workpiece placed inside a wire, and then simulate. Regrettably we find that the bandwidth is very narrow. 
We realize that we have to improve the shape of the workpiece. Firstly we choose to improve it into a shuttle which is cylinder at the middle area and cone at both ends. Unfortunately the results are still found to be not ideal.

Eventually, we change the radius of the inner conductor. At the same time, the ratio of the inner to outer conductor radius is fixed at $1: 2.3$, and the most satisfactory result is obtained.

\section{Calculation And Stimulation}

\subsection{The Influence of Internal and External Radius Ratio}

In the signal transmission area, impedance matching are often required. According to the components connected with the equipment such as RF cable impedance is $50 \Omega$, when the workpiece's wave impedance is $50 \Omega$ or so, minimum loss of signal can be transmitted. Based on transmission line theory, the wave impedance is calculated as

$$
Z_{c}=\frac{60}{\sqrt{\varepsilon_{r}}} \ln \left(\frac{D}{d}\right)
$$

In the equation, $\mathrm{ZC}$ is $50 \Omega$, relative dielectric constant $\varepsilon r$ is 1 (work with air as dielectric), which conduct us the inner and outer diameter of the workpiece is 1:2.3, in future studies, we will take this as a reference ratio.

In order to verify the rationality of the inner and outer radius ratio of $1: 2.3$, we simulate the three parts with inner and outer radius ratios of 1:2.2 (green line) 1:2.3 (red line) and 1:2.4 (blue line).We use XFdtd to obtain Fig.2- 'The S21 parameter map '.

As can be seen from the Fig.2, 1:2.2 (green line) and 1:2.4(blue line) of the signal are reflected a lot while 1:2.3 (red line) of the signal is absorbed by the load so the signal is not reflected a lot due to impedance matching.

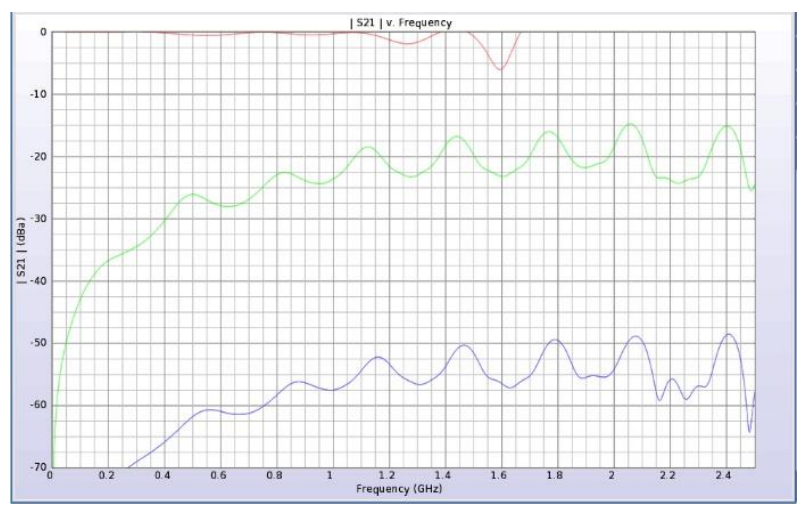

Figure 2. A change in the ratio causes a change in the $S 21$ parameter
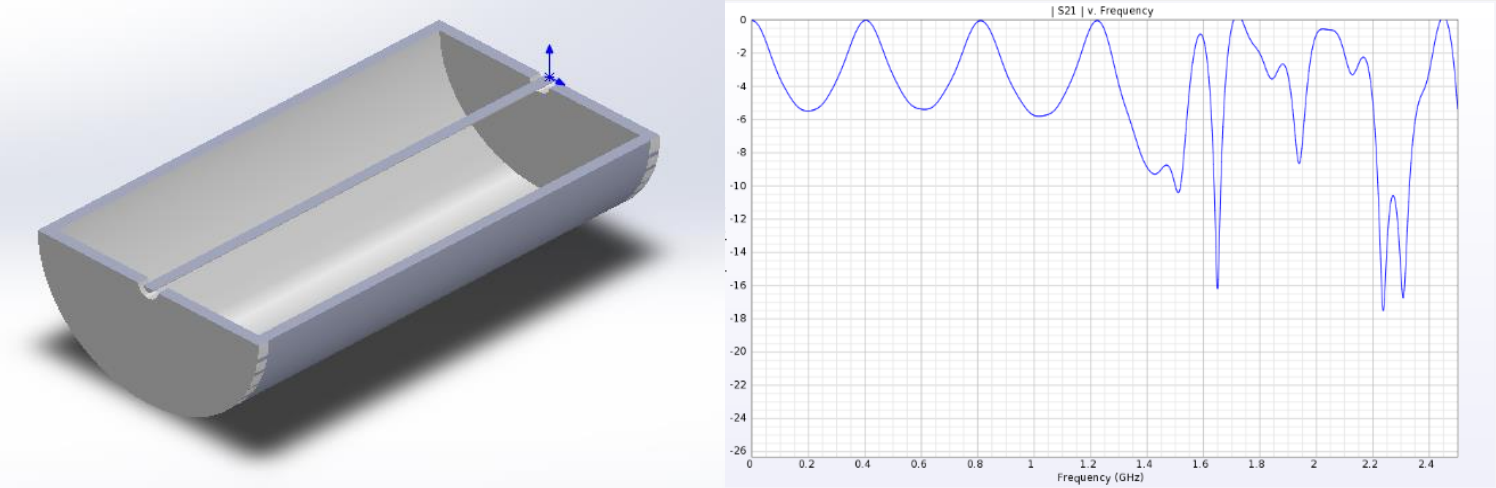

Figure 3.Cylindrical tooling 3D model and its $\mathrm{S} 21$ parameters

\subsection{Influence of Geometric Shapes}

At the beginning we choose the cylinder as the tool (still maintain the ratio of internal and external is 2.3 ) and simulate a $400 \mathrm{~mm}$ tool but the result is very poor with a bandwidth of only $87.5 \mathrm{MHz}$ 

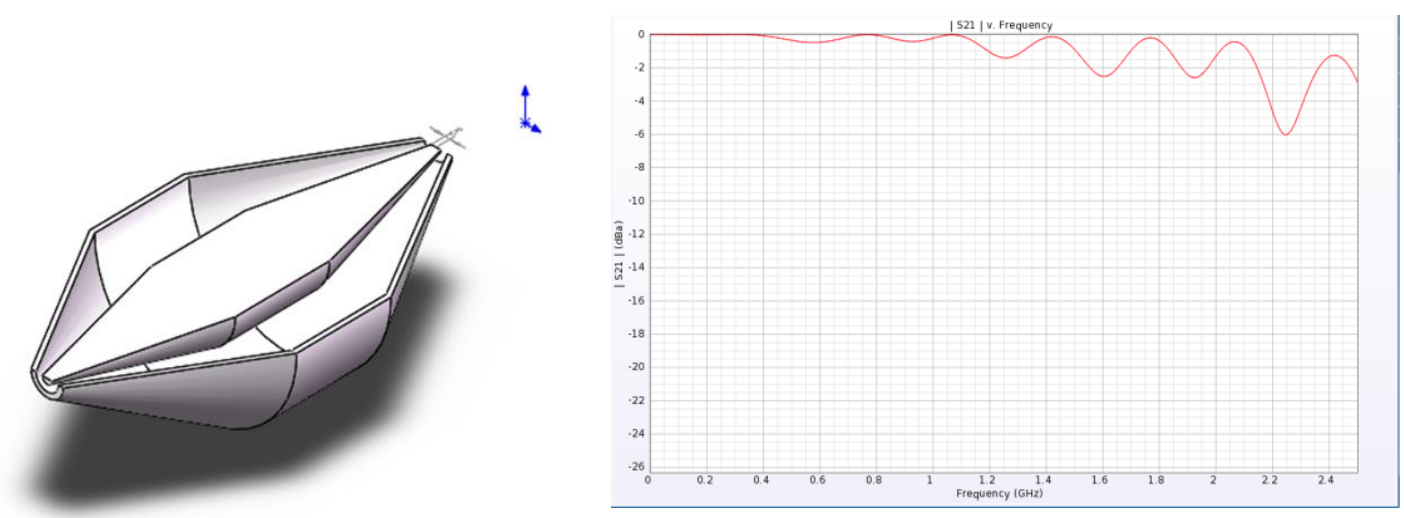

Figure 4.Improved tooling 3D model and its S21 parameters

We guess that its radius ratio at ends of the tool does not match the impedance that reduce the current frequency. Then we improve the shape which taper at both ends. Taking the sensor having much space remaining into account, we increase the middle of the inner conductor radius so that tool is maintained the ratio of 2.3 everywhere. At this point the tool can work up to $2.15 \mathrm{GHz}$ bandwidth.

\subsection{Influence of Tool Length}

After obtaining the best shape of the tool, we try to change the length of the tool and test its impact on the results. We found that the longer the tool is, the wider the band is. In the case of meeting certain bandwidth requirements, the longer the tool is, the smaller the reflection coefficient is in the frequency band $(0-300 \mathrm{MHz})$.

Therefore we arrive at a conclusion that the longer the tool length is, the better the performance is.

\section{Summary}

Based on the results of the simulation and considering the economics of tooling production in the actual situation, we finally determine that the optimum machining parameters of the tool.

The total length of $400 \mathrm{~mm} /$ both inner and outer conductors are fusiform /the radius ratio of $1: 2.3$. This tool band up to $2.15 \mathrm{GHz}$ ideally.

3D renderings and 2D sketches are as follows:
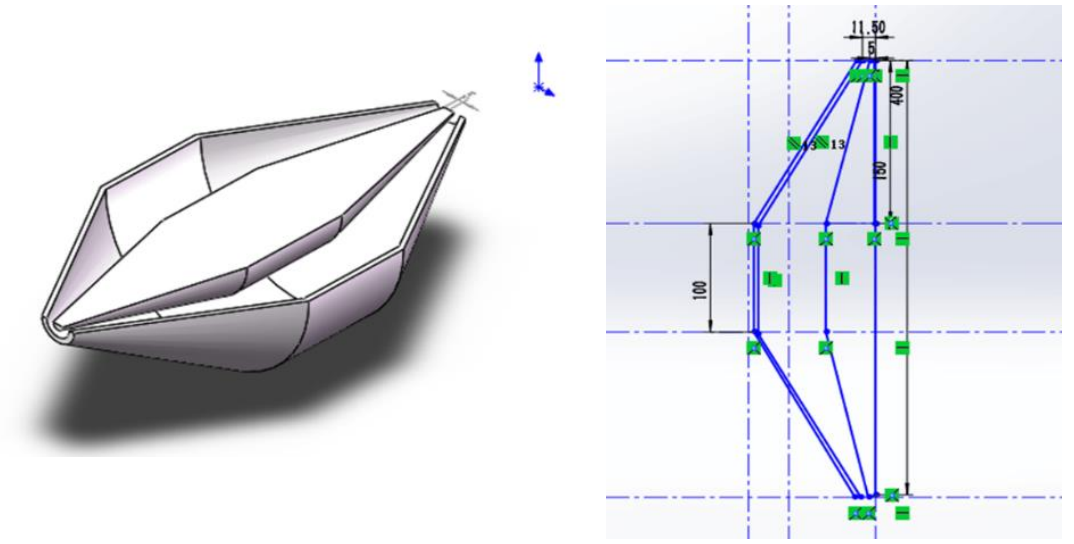

Figure 5. 3D renderings and 2D sketches

\section{Acknowledgements}

The authors gratefully acknowledge the support of the High Voltage Institute in NCEPU. In particular, Master Tang and Master Wong in it give us strong support. 


\section{References}

[1].Ji-Hoon Jang ,Jung-Ik Ha,M. Ohto,K. Ide Seung-Ki Sul, “Analysis of permanent-magnet machine for sensorless control based on high-frequency signal injection", IEEE Transactions on Industry Applications, vol.40,pp. 1595 - 1604,Nov.-Dec. 2004.

[2].Gordon Z. Greenberg, Willard D. Larkin," Frequency-Response Characteristic of Auditory Observers Detecting Signals of a Single Frequency in Noise: The Probe-Signal Method", The Journal of Acoustical Society of America, July 2005, vol.44, pp.1234-1256, Published Online: July 2005.

[3].Christofer Bester, Dona M. P. Jayakody, Geoffrey Hammond, Donald Robertson. "Selective attentional processes in cochlear implant recipients: Measurements of the attentional filter". The Journal of the Acoustical Society of America, 140:6, 4091-4100, online publication date: 2- Dec2016. 